\title{
Projektbericht
}

\section{RenATA Cornejo}

Seit dem 1. Oktober 2018 beteiligen sich drei Universitäten - die Universität der Heiligen Kyrill und Method in Tyrnau (Trnava) als Projektleiter, die Universität Breslau (Wrocław) und die Jan Evangelista Purkyně-Universität in Aussig (Ústí nad Labem) als Projektpartner - an dem dreijährigen Projekt »Internationalisierung und Weiterentwicklung des Doktorandenstudiums « (Erasmus+ 2018-1-SKo1-KA203-046375), welches von der Europäischen Kommission kofinanziert wird. Die Projektidee entstand auf Grund des Bedarfs, die bestehenden Doktorandenstudienprogramme an den drei Universitäten miteinander zu vernetzen, das Lehrveranstaltungsangebot zu erweitern und den Doktorandinnen und Doktoranden aus anderen Ländern ein Auslandssemester anzubieten, welches ihnen an den jeweiligen Universitäten eine entsprechende Anrechnung ermöglicht und somit zur Internationalisierung des Doktorandenstudiums beiträgt.

Ein weiteres Ziel dieser strategischen Partnerschaft zwischen der Slowakei, Tschechien und Polen ist es, das Konzept und Curriculum eines trinationalen Doktorandenstudiums zu entwickeln. Außerdem sollte den beteiligten Lehrkräften durch thematisch ausgerichtete Workshops und gemeinsam erarbeitete Richtlinien eine Stütze für die optimale Betreuung von Doktorandinnen und Doktoranden (nicht nur) aus den Partneruniversitäten angeboten werden. Und schließlich waren Lehrangebote und -materialien zu entwerfen, um nicht nur das Interesse der Studierenden aus den Partneruniversitäten zu wecken, sondern auch sinnvoll zu deren Profilierung beizutragen. Das erwies sich als nicht ganz unproblematisch, da in Tschechien gerade ein neues Hochschulgesetz in Kraft getreten ist, an das die akkreditierten Studienprogramme angepasst werden mussten. Zudem erfuhr in Polen das Hochschulgesetz während des Projekts wesentliche Modifikationen, die u.a. insbesondere das System der Doktorandenschulen beeinflussten. Die erste große Herausforderung war es also, einen begehbaren Weg zu finden und einen gemeinsamen Rahmen für die durchaus unterschiedlichen Doktorandensysteme in Polen und den beiden anderen Ländern zu schaffen, was den Projektbeteiligten schließlich auch gelang. Auf dieser Grundlage konnten ein gemeinsames Curriculum sowie das Lehrveranstaltungsangebot diskutiert und vorbereitet werden.

Das realisierte Projekt, dessen Beendigung im Juni 2021 vorgesehen ist, zielt vor allem auf die Doktorandinnen und Doktoranden der jeweiligen Partneruniversitäten und deren Internationalisierung. So werden im Laufe des Projekts drei internationale Doktorandenkolloquien veranstaltet (das erste in Trnava fand bereits im Juni 2019 statt, das zweite in Ústí nad Labem ist für Herbst 2020 und 
das dritte in Wrocław für Frühjahr 2021 geplant), zu denen ausländische Expertinnen und Experten mit Vorträgen und Workshops eingeladen werden. Dabei wird darauf Wert gelegt, dass die Themen möglichst interdisziplinär angelegt sind, damit sowohl die literaturwissenschaftlich und kulturwissenschaftlich als auch linguistisch interessierten Doktorandinnen und Doktoranden davon möglichst viel profitieren können. Einen integralen Bestandteil dieser Kolloquien bilden das Vorstellen und Diskutieren des eigenen Dissertationsvorhabens bzw. der damit verbundenen Forschungsfragen unter Beteiligung von Professorinnen und Dozenten der jeweiligen Partneruniversität.

Diskutiert wurde unter den Projektpartnern auch die Notwendigkeit, die Doktorandinnen und Doktoranden an das Publizieren von wissenschaftlichen Beiträgen heranzuführen, sie dabei entsprechend zu unterstützen und im Hinblick auf eine geeignete Zeitschrift oder eine andere Publikationsplattform zu beraten, da die Publikationstätigkeit einen wichtigen Bestandteil des Doktorandenstudiums in allen drei Ländern bildet. Dabei entstand die Idee, eine neue Schriftenreihe ins Leben zu rufen, die den ausländischen Doktorandinnen und Doktoranden die Möglichkeit bieten würde, »ihre Forschungsfragen, Problemstellungen und erste Forschungsansätze und -ergebnisse einer interessierten Öffentlichkeit zu präsentieren und damit zur Diskussion zu stellen « (Demčišák/Horňáček Banášová 2020: 8). Die neu gegründete Publikationsplattform »Doktorandenforum Auslandsgermanistik « soll dazu beitragen, die ausländischen Doktorandinnen und Doktoranden stärker als Nachwuchswissenschaftler im deutschsprachigen Raum wahrzunehmen, was durch die Herausgabe im renommierten Leipziger Universitätsverlag gewährleistet werden soll. Die Schriftenreihe versteht sich als »eine Plattform, die Nachwuchswissenschaftlerinnen und Nachwuchswissenschaftlern aus allen Bereichen der Germanistik (Sprachwissenschaft, Literaturwissenschaft, Deutsch-Didaktik, Deutsch als Fremdsprache) offensteht« (ebd.). Dabei sollen unterschiedliche Formen der Forschung Berücksichtigung finden - Beiträge von Doktorandenkolloquien und -konferenzen, Sammelbände, kollektiv oder selbstständig erarbeitete Monographien. Die wissenschaftliche Qualität garantiert der Wissenschaftliche Beirat der Schriftenreihe, für den Wissenschaftlerinnen und Wissenschaftler aus Deutschland, Österreich, den drei beteiligten Partnerländern sowie aus Ungarn, Griechenland, Italien, Spanien und Estland gewonnen werden konnten.

Der erste Band, mit dem diese Schriftenreihe eröffnet wird, ist unter dem Titel »Germanistische Forschungsfragen in Trnava, Ústí nad Labem und Wrocław I.« (hg. v. Jan Demčišák und Monika Horňáček Banášová) 2020 im Leipziger Universitätsverlag erschienen, im Frühjahr 2021 ist die Herausgabe des zweiten Bandes geplant - beide präsentieren die Forschungsergebnisse von Doktorandinnen und Doktoranden der am Projekt beteiligten Universitäten. Die Schriftenreihe ist jedoch für alle Interessierten offen - entsprechend ihrer Intention liegt es den Herausgeberinnen daran, den Nachwuchswissenschaftlern der sog. Auslandsgermanistik eine geeignete Publikationsplattform anzubieten und durch die künftige Herausgabe von inspirierenden Bänden zu einem fruchtba- 
ren wissenschaftlichen Austausch unter den Germanistinnen und Germanisten im In- und Ausland beizutragen.

Nähere Informationen zum Projekt sind in slowakischer, deutscher und englischer Sprache auf den folgenden Internet-Seiten zu finden:

- http://kger.ff.ucm.sk/sk/projekty-a-granty/

- https://www.erasmusplus.sk/index.php?sw=33\&menu=12\&proj=2018-1SKo1-KA203-046375

- https://ec.europa.eu/programmes/erasmus-plus/projects/eplus-projectdetails/\#project/2018-1-SKo1-KA203-046375

Nähere Informationen zur Schriftenreihe »Doktorandenforum Auslandsgermanistik« in deutscher Sprache (hier steht auch der vollständige Text des ersten Bandes zur Verfügung) können hier entnommen werden: http://ff.ujep.cz/ publikationen/66-de/9644-doktorandenforum-auslandsgermanistik-de.

\section{LITERATUR}

Demčišák, Jan/Horňáček Banášová, Monika (Hg; 2020): Germanistische Forschungsfragen in Trnava, Ústí nad Labem und Wrocław I (= Doktorandenforum Auslandsgermanistik, Bd. 1). Leipzig. 
